\title{
Using AUSGeoid2020 and its error grids in surveying computations
}

\author{
W.E. Featherstone ${ }^{1 *}$, J.C. McCubbine ${ }^{2}$, S.J. Claessens ${ }^{1}$, D. Belton ${ }^{1}$, N.J. Brown ${ }^{2}$ \\ 1 School of Earth and Planetary Sciences, Curtin University, GPO Box U1987, Perth, WA 6845, Australia \\ 2 National Geodesy Section, Geoscience Australia, GPO Box 378, Canberra, ACT 2601, Australia
}

ORCIDs: W.E. Featherstone (http://orcid.org/0000-0001-9644-4535), J.C. McCubbine

(http://orcid.org/0000-0002-6939-1340), S.J. Claessens (http://orcid.org/0000-0003-4935-6916),

D. Belton (http://orcid.org/0000-0002-2879-7918), N.J. Brown (http://orcid.org/0000-0001-9476-974X)

\begin{abstract}
AUSGeoid2020 provides the separation between the GRS80 ellipsoid used with the Geocentric Datum of Australia 2020 (GDA2020) and the Australian Height Datum (AHD), as well as vertical deflections/deviations of the local gravity vector from the GRS80 ellipsoidal normal. The AUSGeoid2020 GDA2020-AHD separations are accompanied by location-specific uncertainties propagated from the various input data. Here we present location-specific uncertainties for the vertical deflections derived from the horizontal gradients of the AGQG2017 gravimetric-only quasigeoid model, upon which AUSGeoid2020 is based. We also present summarised formulas and worked examples for the propagation of geoid and vertical deflection errors through some common geodetic surveying computations, as well as a demonstration of their effects on least squares adjustments of small simulated geodetic networks.
\end{abstract}

\section{Introduction}

The adoption of the Geocentric Datum of Australia 2020 (GDA2020) (ICSM, 2018) has necessitated recomputation of a compatible version of AUSGeoid2020 (Brown et al., 2018a,b) so that height transformations account for the $\sim 90 \mathrm{~mm}$ change in ellipsoidal heights from GDA94, as well as profiting from more and newer data. AUSGeoid2020 delivers Australian Height Datum (AHD) heights more directly from GNSS surveys (cf. Featherstone, 1998). Like its predecessor, AUSGeoid09 (Brown et al., 2011; Featherstone et al, 2011), AUSGeoid2020 has fitted the AGQG2017 gravimetric-only quasigeoid model (Featherstone et al., 2018) to 7,500 GPS-AHD heights across the continent using least squares prediction (Brown et al., 2018a,b). AUSGeoid2020 is also accompanied by a grid of vertical deflections/deviations computed from the horizontal gradients of AGQG2017.

Australia's move to geocentric datums means that approximations in geodetic surveying data reduction that were previously acceptable for the non-geocentric Australian Geodetic Datums AGD66 and AGD84 (because the Australian National Spheroid was aligned to the Australian geoid (Mather, 1970)) are no longer acceptable, particularly for geodetic surveying, as follows. The so-called "sea level correction" now has to include the GDA2020-AHD separations (e.g., Featherstone, 1997) to properly reduce slope distances to the GRS80 ellipsoid, else a $1 \mathrm{ppm}$ horizontal scale error will be introduced for every $6.35 \mathrm{~m}$ of omitted AHD-GRS80 separation. Vertical deflections have generally increased, so now have to be considered in the reduction of several types of terrestrial surveying observations to the GRS80 ellipsoid that is used by GDA2020 and AUSGeoid2020 (e.g., Featherstone and Rüeger, 2000).

Unlike all its predecessors that used a "one size fits all" uncertainty estimate for the entire continent, AUSGeoid2020 GDA2020-AHD separations are now accompanied by a location-specific error grid at a 1'x1' spacing (Brown et al., 2018a). AUSGeoid2020 is public-domain and available at https://s3ap-southeast-2.amazonaws.com/geoid/. It is important to use the latest version number (AUSGeoid2020_20180201.gsb at time of writing) because a patch has been applied to account for erroneous input data near Melbourne (Brown et al., 2018a, Appendix B), and other patched updates may be necessary in future. The NTV2 format (.gsb file extension) can be used with the public-domain GeoidInt software, available at https://www.icsm.gov.au/sites/default/files/Geoidlnterpolation 0.zip. Alternatively, an on-line computation tool is at http://www.ga.gov.au/ausgeoid/nvalcomp.jsp to determine GDA2020AHD separations and vertical deflections. 


\section{Using AUSGeoid2020 GDA2020-AHD separations}

The most common application of AUSGeoid2020 is the transformation of GNSS-derived ellipsoidal heights to AHD heights, or vice versa. This can be done in an absolute sense or a relative sense over GNSS baselines (e.g., Featherstone, 2008).

First, some minor technical clarifications: AUSGeoid2020 is strictly neither a geoid model nor a quasigeoid model. The computation of AGQG2017 delivered a gravimetric-only quasigeoid model, but this was subsequently tilted (cf. Featherstone and Filmer, 2012) and surface-fitted to GPS-AHD heights across the continent using least squares prediction (Brown et al., 2018a). As such, its physical-geodetic definition in terms of the Earth's gravity field is somewhat nebulous; instead, it gives a model of the separation between the AHD and GRS80 ellipsoid (cf. Featherstone, 1998), realised as GDA2020. In the following, we use the symbol $N$ to describe the AUSGeoid2020 GDA2020-AHD separation solely because this symbology is more familiar to GNSS users for height determination, but use $\zeta$ when referring to AGQG2017 (Appendix A and Section 2.2). Featherstone and Kuhn (2006) review these subtleties of heights in the Australian context.

\subsection{Absolute AHD height determination}

This refers to when a single GNSS receiver is used and data processed using tools such as AUSPOS (http://www.ga.gov.au/bin/gps.pl), precise point positioning (PPP), or commercially available real-time kinematic (RTK) corrections. The transformation between a GNSS ellipsoidal height and derived AHD height is simple, but an approximation is made because the AHD height is not measured along the ellipsoidal normal. Taking the mean vertical deflection over the Australian mainland as 6" (Featherstone and Rüeger, 2000) and the mean height as $350 \mathrm{~m}$ (e.g., Hirt et al., 2010), this approximation is less than a micron. Taking an extreme of a maximum vertical deflection of 50" (Appendix A) and the $2228 \mathrm{~m}$ height of Mount Kosciusko, this approximation remains less than $0.1 \mathrm{~mm}$. As such, full equality is used in Eq. (1).

An AHD height $(H)$ is derived from a measured absolute GNSS ellipsoidal height $(h)$ using the AUSGeoid2020 AHD-GRS80 separation $(N)$ at that point

$H=h-N$

The corresponding error is given by Eq. (2), which also includes the uncertainty $\sigma^{2}\left(H_{A}\right)$ in the usermeasured antenna height of the antenna reference point (ARP) above the physical ground mark. $\sigma(H)=\sqrt{\sigma^{2}(h)+\sigma^{2}(N)+\sigma^{2}\left(H_{A}\right)}$

where the covariances among $h, N$ and $H_{A}$ are zero because they are all independent.

The following worked example uses the ellipsoidal height from a six-hour GPS occupation of an AHD benchmark in Perth, Western Australia. The GPS data were post-processed in AUSPOS. The 95\%confidence errors reported by AUSPOS have been scaled to one standard deviation by dividing by 1.96 for a univariate distribution. Note that the uncertainty grid with AUSGeoid2020 is one standard deviation.

\begin{tabular}{|l|r|r|}
\hline \multirow{2}{*}{ GDA2020 geodetic coordinates } & $\phi=-31^{\circ} 51^{\prime} 28.66464^{\prime \prime}$ & \\
\cline { 2 - 3 } & $\lambda=115^{\circ} 46^{\prime} 06.55560^{\prime \prime}$ & \\
\cline { 2 - 3 } & $h=-3.047 \mathrm{~m}$ & $\sigma(h)= \pm 0.018 \mathrm{~m}$ \\
\hline AUSGeoid2020 AHD-GRS80 separation & $N=-32.990 \mathrm{~m}$ & $\sigma(N)= \pm 0.082 \mathrm{~m}$ \\
\hline AUSGeoid2020-derived AHD Height & $H=29.943 \mathrm{~m}$ & $\sigma(H)= \pm 0.084 \mathrm{~m}$ \\
\hline Published AHD height (from Landgate) & $H=29.987 \mathrm{~m}$ & \\
\hline
\end{tabular}

Table 1: Worked example of absolute AHD height determination from GNSS (Eq. 1), error propagation via Eq. (2), and a comparison with a published AHD height from differential levelling.

As a check, the on-line AUSGeoid2020 calculator http://www.ga.gov.au/ausgeoid/nvalcomp.jsp was used, which gave identical values. As the GNSS-measured ellipsoidal height and AUSGeoid2020 are independent, SLOPOV can be used to give the error in the derived AHD height: $\sqrt{0.018^{2}+0.082^{2}}=0.084 \mathrm{~m}$. This value is slightly different to that reported by AUSPOS $(0.085 \mathrm{~m})$, but only because three decimal places 
have been carried in this check calculation. The uncertainty in the measurement of the GNSS antenna height (say, $1 \mathrm{~mm}$ ) can also be added, again using independence: $\sqrt{0.085^{2}+0.001^{2}}=0.085 \mathrm{~m}$, but which is insignificant in relation to the dominating uncertainty coming from the AUSGeoid2020 error grid. When considering the uncertainty in the GNSS-derived AHD height, it agrees statistically with the published AHD height (Table 1).

Correct measurement and identification of the GNSS antenna's height is probably one of the more common blunders made when surveying with GNSS. There are two main factors that can come into play: (i) mistakes in or complete omission of the measured height of the ARP above the physical ground mark; and (ii) incorrect identification of the antenna type (make and model) used during data processing. Most geodetic-grade GNSS antennas have been calibrated by the International GNSS Service (IGS) (Dow et al., 2009) and stored in a so-called .atx file (ftp://igs.org/pub/station/general/antex14.txt). This accounts for antenna phase centre offsets and elevation- and azimuth-dependent phase centre variations, hence GNSS antennas should always be oriented north.

To gauge the effect of using an incorrect antenna type on GNSS-measured ellipsoidal heights, the above GPS data were processed in AUSPOS using an [incorrect] TRIM59800.00 DOME antenna instead of the [correct] LEIC15 NONE antenna. The reported GDA2020 ellipsoidal height changed to $-2.845 \pm 0.020 \mathrm{~m}$ and the AUSGeoid2020-derived AHD height changed to $30.145 \pm 0.086 \mathrm{~m}$. When comparing this to the published AHD height of the benchmark (29.987 m; Table 1), the results are inconsistent. As such, the statistics for the solution are incorrect and do not alert one to the actual error. Therefore, great care must be taken over antenna heights and types when using GNSS for height determination.

\subsection{Relative AHD height determination}

This refers to when two or more GNSS receivers are used and processed in relative carrier-phase mode to deliver ellipsoidal height differences over baselines. An AHD height difference $(\Delta H)$ is derived from a relative GNSS ellipsoidal height difference $(\Delta h)$ using the difference in AUSGeoid2020 AHD-GRS80 separation $(\Delta N)$ at each end of the baseline:

$\Delta H_{1,2}=\Delta h_{1,2}-\Delta N_{1,2}$

However, the simple use of AUSGeoid2020 values at each end of the GNSS baseline neglects the benefit of using relative GNSS so that common-mode errors cancel, not only in the GNSS baseline processing, but also in the AUSGeoid2020 separation difference, thus making the derived height difference more precise (Kearsley, 1986, 1988).

Brown et al. (2018a) thus devised an exponential decorrelation function from $\sim 8,000$ GPS-AHD stations not used in the production of AUSGeoid2020 to account for this. The error propagation when using Eq. (3) for relative GNSS heighting becomes Eq. (11) from Brown et al. (2018a), noting the change in symbology for the present manuscript:

$$
\sigma\left(\Delta H_{1,2}\right)=\sqrt{\sigma^{2}\left(h_{1}\right)+\sigma^{2}\left(h_{2}\right)-2 \operatorname{cov}\left(h_{1}, h_{2}\right)+\left\{\sigma^{2}\left(N_{1}\right)+\sigma^{2}\left(N_{2}\right)\right\}\left(1-k \exp ^{-3 l / a}\right)}
$$

where, all for one-sigma, $\sigma\left(\Delta H_{1,2}\right)$ is the uncertainty in the GNSS-derived AHD height difference, $\sigma^{2}(h)$ is the GNSS-software-provided ellipsoidal height error variance at each end of the baseline, $\operatorname{cov}\left(h_{1}, h_{2}\right)$ is the GNSS-software-provided covariance for that baseline, $\sigma^{2}(N)$ is the AUSGeoid2020 uncertainty at each end of the baseline, $l$ is the baseline length in metres, and the empirically determined parameters for the error decorrelation function $\left(1-k \exp ^{-3 l / a}\right)$ are $k=0.68$ and $a=63,151 m$ (Brown et al., 2018a). By GNSSsoftware-provided, we mean the values delivered directly by the GNSS processing software, taken at face value, but acknowledging they may be overoptimistic (e.g., Rothacher, 2002).

The worked example below is taken directly from Brown et al. (2018b) for a $7.5 \mathrm{~km}$-long GPS baseline. The value of $\sigma\left(\Delta H_{1,2}\right)$ is $102 \mathrm{~mm}$ when the decorrelation function is considered, whereas it would be a larger $141 \mathrm{~mm}$ should this not be considered. 
- With decorrelation and GNSS baseline covariance:

$\sigma\left(\Delta H_{1,2}\right)=\sqrt{\sigma^{2}\left(h_{1}\right)+\sigma^{2}\left(h_{2}\right)-2 \operatorname{cov}\left(h_{1}, h_{2}\right)+\left\{\sigma^{2}\left(N_{1}\right)+\sigma^{2}\left(N_{2}\right)\right\}\left(1-k_{\exp ^{-3 l} / a}\right)}$
$\sigma\left(\Delta H_{1,2}\right)=\sqrt{0.0023^{2}+0.0023^{2}-2\left(4.352 \times 10^{-6}\right)+\left\{0.100^{2}+0.099^{2}\right\}\left(1-0.68 \exp ^{-3 \times 7500 / 63151}\right)}=0.102 \mathrm{~m}$

- Without decorrelation and GNSS baseline covariance:

$\sigma\left(\Delta H_{1,2}\right)=\sqrt{\sigma^{2}\left(h_{1}\right)+\sigma^{2}\left(h_{2}\right)+\sigma^{2}\left(N_{1}\right)+\sigma^{2}\left(N_{2}\right)}$

$\sigma\left(\Delta H_{1,2}\right)=\sqrt{0.0023^{2}+0.0023^{2}+0.100^{2}+0.099^{2}}=0.141 \mathrm{~m}$

GDA2020 geodetic coordinates of station 1 and standard deviations from GNSS processing software

\begin{tabular}{|l|r|r|}
\cline { 2 - 3 } & $h_{1}=340.586 \mathrm{~m}$ & $\sigma\left(h_{1}\right)= \pm 0.002(3) \mathrm{m}$ \\
\hline \multirow{2}{*}{$\begin{array}{l}\text { GDA2020 geodetic coordinates of station 2 and } \\
\text { standard deviations from GNSS processing software }\end{array}$} & $\phi_{2}=-26.292780$ & \\
\cline { 2 - 3 } & $\lambda_{2}=148.485857$ & \\
\cline { 2 - 3 } & $h_{2}=352.494 \mathrm{~m}$ & $\sigma\left(h_{2}\right)= \pm 0.002(3) \mathrm{m}$ \\
\hline Covariance from GNSS processing software & & $\operatorname{cov}\left(h_{1}, h_{2}\right)=4.352 \times 10^{-6}$ \\
\hline AUSGeoid2020 GDA2020-AHD separation of Site 1 & $N_{1}=38.754 \mathrm{~m}$ & $\sigma\left(N_{1}\right)= \pm 0.100 \mathrm{~m}$ \\
\hline AUSGeoid2020 GDA2020-AHD separation of Site 2 & $N_{2}=38.868 \mathrm{~m}$ & $\sigma\left(N_{2}\right)= \pm 0.099 \mathrm{~m}$ \\
\hline $\begin{array}{l}\text { AUSGeoid2020-derived AHD height difference (Eq. 3) } \\
\text { and relative error (Eq.5) }\end{array}$ & $\Delta H_{1,2}=-11.794 \mathrm{~m}$ & $\sigma\left(\Delta H_{1,2}\right)= \pm 0.102 \mathrm{~m}$ \\
\hline
\end{tabular}

Table 2: Worked example of relative AHD height determination from GNSS and error propagation with Eq. (5).

In short, observation of GNSS baselines remains preferable should one want to determine more precise AHD height differences from GNSS (cf. Kearsley, 1986, 1988). Brown et al. (2018a) also show that GNSS-AUSGeoid2020-derived AHD height differences are inferior to Australian class LC differential levelling (allowable misclose of $12 \mathrm{~mm}$ per square-root of distance between stations) over distances less than $\sim 3 \mathrm{~km}$. As such, we should not totally dispose of our differential levelling instruments quite just yet.

The error grid that accompanies AUSGeoid2020 GDA2020-AHD separations results from a linear combination of the errors from AGQG2017 (Featherstone et al., 2018), the published AHD heights and the GDA2020 ellipsoidal heights (Brown et al., 2018a); cf. Eq. (2). This was deliberate so as to account for errors originating from all data sources contributing to AUSGeoid2020. These were computed at all $\sim 7,500$ points scattered across the continent (Featherstone et al., 2018, Figure $6 \mathrm{~d}$ ) then gridded at a $1^{\prime} \times 1^{\prime}$ resolution using the same covariance parameters for the least squares prediction used to generate AUSGeoid2020. This has caused the errors in AUSGeoid2020 to be, on average, $\sim 40 \mathrm{~mm}$ larger than the errors from AGQG2017 (cf. Featherstone et al., 2018, Section 2.4).

For instance, if using AGQG2017 and its error grid instead of the AUSGeoid2020 values in Table 2 and Eq. (6), gives: $\zeta_{1}=38.687 \mathrm{~m}, \zeta_{2}=38.802 \mathrm{n}, \sigma\left(\zeta_{1}\right)= \pm 0.057 \mathrm{~m}, \sigma\left(\zeta_{2}\right)= \pm 0.057 \mathrm{~m}, \Delta H_{1,2}^{*}=-11.793 \mathrm{~m}$, $\sigma\left(\Delta H_{1,2}^{*}\right)= \pm 0.081 \mathrm{~m}$. No decorrelation function has been applied for correlated errors in AGQG2017, so the relative error is probably even smaller than calculated here. Although the GNSS-AGQG2018-derived height difference is essentially the same as that from AUSGeoid2020 (11.794 m; Table 2), it is still essential to clearly document which model is used in the transformation so as to avoid ambiguity and confusion, especially over longer baselines where the difference between AGQG2017 and AUSGeoid2020 will be larger than this case.

It is entirely plausible that the relative error in AUSGeoid2020 is less than that calculated in Tables 2 and 9, even after application of the decorrelation function, especially for very short baselines. One reason is that very few baselines less than $5 \mathrm{~km}$ were used to determine the decorrelation function in Brown et al. 
(2018a). Another reason is that we set the parameter $k=0.68$. As such, this remains a subject for future scrutiny so as to better ascertain over what distances GNSS and AUSGeoid2020 really is superior to differential levelling.

\subsection{Reduction of electronic distance ( $l$ ) measurements (EDM) to the ellipsoid ( $s$ )}

Featherstone (1997) showed that the GDA2020-AHD separation is needed in this reduction, whereas it could usually be neglected with the AGD66/84 in the so-called "sea level correction". Featherstone and Rüeger (2000) refined it to consider changes in geoid height along the baseline. Here, we now consider the uncertainty in the AUSGeoid2020 GDA2020-AHD separations on this surveying data reduction. The AHD height difference can be determined by differential levelling or trigonometric heighting.

In this simplified example, we consider propagation of errors from the AUSGeoid2020 GDA2020AHD separation. From Rüeger (1996) and Featherstone and Rüeger (2000, Eq. 29) with changed symbology:

$\sigma(s)= \pm\left\|\Delta h_{1,2}\right\|\left(\frac{\sigma\left(\Delta N_{1,2}\right)}{l}\right)$

where $s$ is the geodesic distance on the ellipsoid, $l$ is the EDM-measured slope distance, and from Eq. (5) including the error decorrelation:

$\sigma\left(\Delta N_{1,2}\right)=\sqrt{\left\{\sigma^{2}\left(N_{1}\right)+\sigma^{2}\left(N_{2}\right)\right\}\left(1-k \exp ^{-3 l / a}\right)}$

Using the values from Table 2 for an EDM-measured slope distance of $l=7,501.586 \mathrm{~m}$ (reverse-engineered from the ellipsoidal distance using similar triangles) gives an error of $\pm 0.2 \mathrm{~mm}$, which is insignificant in relation to the precision of even the most precise EDM instruments.

\section{Using AUSGeoid2020 vertical deflections}

Here, we revisit the corrections for vertical deflections in the prime vertical $\mathrm{EW}(\eta)$ and meridian NS $(\xi)$ as given by Featherstone and Rüeger (2000), but this time accounting for uncertainties in the vertical deflections. Appendix A describes the computation of new location-specific deflection uncertainties derived from the horizontal gradients of AGQG2017. Strictly, these are Pizzetti deflections (cf. Jekeli, 1999), whereas Helmert deflections at the Earth's surface are needed for the reduction of terrestrial geodetic surveying observations to the ellipsoid (cf. Featherstone and Rüeger 2000), but the difference is postulated to be around 1" (arc-second). In the following examples, the Pizzetti deflections calculated from AGQG2017 are assumed to be Helmert deflections as the formally propagated uncertainty is greater than their postulated conceptual difference.

As well as replicating the correction/reduction formulas from Featherstone and Rüeger (2000), we derive some new error propagation formulas under the following assumptions: (i) throughout, uncertainties in the two deflection components have been taken to be independent following the result of Eq. (A9) in Appendix A; (ii) the uncertainty in the geodetic latitude $\phi$, deflections $(\xi, \eta)$, gyrotheodolite / astrogeodetic azimuth $(A)$, measured zenith angle $(Z)$ and measured horizontal directions $(D)$ are all assumed to be independent; and (iii) astronomical coordinates $\Lambda, \Phi$ and deflections are all considered to be mutually independent. Errors in the measurement of instrument height, target height and centring are assumed small in relation to the errors emanating from the vertical deflections, so neglected from the formulas presented. The following worked examples use the below coordinates, AUSGeoid2020 vertical deflections and their uncertainties bi-cubically interpolated from the location-specific error grid described in Appendix A.

\begin{tabular}{|l|r|r|}
\hline & & Uncertainty \\
\hline GDA2020 geodetic coordinates & $\phi=-25^{\circ} 56^{\prime} 56.86000^{\prime \prime}$ & -- \\
\hline & $\lambda=133^{\circ} 12^{\prime} 37.21000^{\prime \prime}$ & -- \\
\hline AUSGeoid2020 vertical deflections & $\xi=+2.44^{\prime \prime}$ & $\sigma(\xi)= \pm 2.85^{\prime \prime}$ \\
\hline & $\eta=-7.96 "$ & $\sigma(\eta)= \pm 3.11^{\prime \prime}$ \\
\hline
\end{tabular}


Importantly, in order to perform/replicate the error propagation in all the worked examples in the remainder of this Section, it is necessary that $\xi$ and $\eta$ are used in radian measure, the result after applying the square root converted back to arc-seconds.

\subsection{Reduction of measured zenith angles $(Z)$ to the ellipsoid $(z)$ in geodetic azimuth $(\alpha)$}

$z=Z-(\xi \cdot \cos (\alpha)+\eta \cdot \sin (\alpha))$

$\sigma(z)=\sqrt{\sigma^{2}(Z)+\cos ^{2}(\alpha) \cdot \sigma^{2}(\xi)+\sin ^{2}(\alpha) \cdot \sigma^{2}(\eta)+(\eta \cdot \cos (\alpha)-\xi \cdot \sin (\alpha))^{2} \cdot \sigma^{2}(\alpha)}$

\begin{tabular}{|c|c|c|r|r|r|}
\hline $\begin{array}{c}\text { Measured } \\
\text { zenith } \\
\text { angle }(Z)\end{array}$ & $\begin{array}{c}\text { Uncertainty } \\
\sigma(Z)\end{array}$ & $\begin{array}{c}\text { Geodetic } \\
\text { azimuth } \\
\text { of line }(\alpha)\end{array}$ & $\begin{array}{c}\text { Uncertainty } \\
\sigma(\alpha)\end{array}$ & $\begin{array}{c}\text { Geodetic } \\
\text { zenith } \\
\text { angle }(z)\end{array}$ & $\begin{array}{c}\text { Uncertainty } \\
\sigma(z)\end{array}$ \\
\hline $45^{\circ} 00^{\prime} 00^{\prime \prime}$ & $\pm 2^{\prime \prime} 00^{\circ} 00^{\prime} 00^{\prime \prime}$ & $\pm 1^{\prime \prime} 44^{\circ} 59^{\prime} 57.56^{\prime \prime}$ & $\pm 3.48^{\prime \prime}$ \\
\hline $85^{\circ} 00^{\prime} 00^{\prime \prime}$ & $\pm 2^{\prime \prime} 45^{\circ} 00^{\prime} 00^{\prime \prime}$ & $\pm 1^{\prime \prime} 85^{\circ} 00^{\prime} 03.90^{\prime \prime}$ & $\pm 3.59^{\prime \prime}$ \\
\hline $89^{\circ} 00^{\prime} 00^{\prime \prime}$ & $\pm 2^{\prime \prime} 90^{\circ} 00^{\prime} 00^{\prime \prime}$ & $\pm 1^{\prime \prime} 89^{\circ} 00^{\prime} 07.96^{\prime \prime}$ & $\pm 3.70^{\prime \prime}$ \\
\hline $45^{\circ} 00^{\prime} 00^{\prime \prime}$ & $\pm 0^{\prime \prime} 00^{\circ} 00^{\prime} 00^{\prime \prime}$ & $\pm 0^{\prime \prime} 44^{\circ} 59^{\prime} 57.56^{\prime \prime}$ & $\pm 2.85^{\prime \prime}$ \\
\hline $85^{\circ} 00^{\prime} 00^{\prime \prime}$ & $\pm 0^{\prime \prime} 45^{\circ} 00^{\prime} 00^{\prime \prime}$ & $\pm 0^{\prime \prime} 85^{\circ} 00^{\prime} 03.90^{\prime \prime}$ & $\pm 2.98^{\prime \prime}$ \\
\hline $89^{\circ} 00^{\prime} 00^{\prime \prime}$ & $\pm 0^{\prime \prime} 90^{\circ} 00^{\prime} 00^{\prime \prime}$ & $\pm 0^{\prime \prime} 89^{\circ} 00^{\prime} 07.96^{\prime \prime}$ & $\pm 3.11^{\prime \prime}$ \\
\hline
\end{tabular}

Table 3: Worked examples of reducing measured zenith angles to geodetic zenith angles (Eq. 9) and error propagation (Eq. 10) using the deflection errors from Appendix A. The [unrealistic] cases of zero observational error are included to assist gauge the relative contribution of the deflection errors.

\subsection{Reduction of measured horizontal directions $(D)$ to the ellipsoid $(d)$ in geodetic azimuth $(\alpha)$}

This worked example uses the errors propagated from the measured zenith angles in Table 3 . The [small $<<1$ ] skew normal correction has also been neglected.

$d=D-(\xi \cdot \sin \alpha-\eta \cdot \cos \alpha) \cot z$

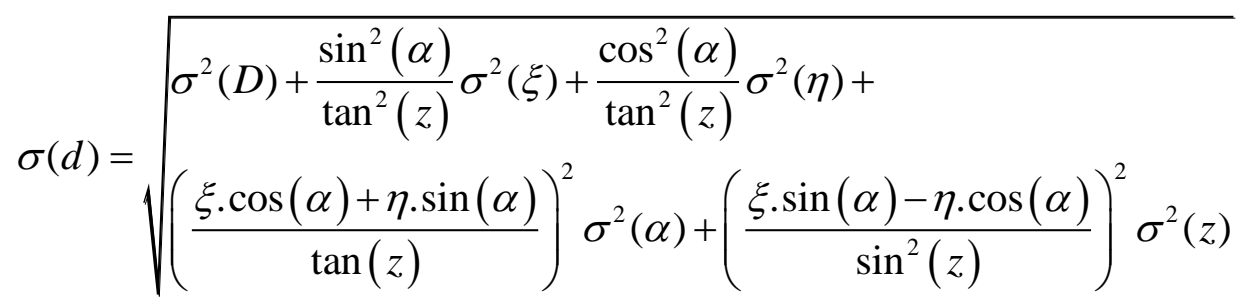

\begin{tabular}{|c|r|r|r|r|r|r|r|}
\hline $\begin{array}{c}\text { Measured } \\
\text { horizontal } \\
\text { direction }(D)\end{array}$ & $\begin{array}{c}\text { Uncertainty } \\
\sigma(D)\end{array}$ & $\begin{array}{c}\text { Geodetic } \\
\text { zenith } \\
\text { angle }(z)\end{array}$ & $\begin{array}{c}\text { Uncertainty } \\
\sigma(z)\end{array}$ & $\begin{array}{r}\text { Geodetic } \\
\text { azimuth } \\
\text { of line }(\alpha)\end{array}$ & $\begin{array}{r}\text { Uncertainty } \\
\sigma(\alpha)\end{array}$ & $\begin{array}{c}\text { Reduced } \\
\text { horizontal } \\
\text { direction }(d)\end{array}$ & $\begin{array}{r}\text { Uncertainty } \\
\sigma(d)\end{array}$ \\
\hline $45^{\circ} 00^{\prime} 00.00^{\prime \prime}$ & $\pm 1^{\prime \prime} 44^{\circ} 59^{\prime} 57.56^{\prime \prime}$ & $\pm 3.48^{\prime \prime}$ & $45^{\circ}$ & $\pm 1^{\prime \prime} 44^{\circ} 59^{\prime} 52.65^{\prime \prime}$ & $\pm 3.15^{\prime \prime}$ \\
\hline $45^{\circ} 00^{\prime} 00.00^{\prime \prime}$ & $\pm 1^{\prime \prime} 85^{\circ} 00^{\prime} 03.90^{\prime \prime}$ & $\pm 3.59^{\prime \prime}$ & $45^{\circ}$ & $\pm 1^{\prime \prime}$ & $44^{\circ} 59^{\prime} 59.36^{\prime \prime}$ & $\pm 1.03^{\prime \prime}$ \\
\hline $45^{\circ} 00^{\prime} 00.00^{\prime \prime}$ & $\pm 1^{\prime \prime} 89^{\circ} 00^{\prime} 07.96^{\prime \prime}$ & $\pm 3.69^{\prime \prime}$ & $45^{\circ}$ & $\pm 1^{\prime \prime}$ & $44^{\circ} 59^{\prime} 59.87^{\prime \prime}$ & $\pm 1.00^{\prime \prime}$ \\
\hline $45^{\circ} 00^{\prime} 00.00^{\prime \prime}$ & $\pm 0^{\prime \prime} 44^{\circ} 59^{\prime} 57.56^{\prime \prime}$ & $\pm 0^{\prime \prime}$ & $45^{\circ}$ & $\pm 0^{\prime \prime} 44^{\circ} 59^{\prime} 52.65^{\prime \prime}$ & $\pm 2.98^{\prime \prime}$ \\
\hline $45^{\circ} 00^{\prime} 00.00^{\prime \prime}$ & $\pm 0^{\prime \prime} 85^{\circ} 00^{\prime} 03.90^{\prime \prime}$ & $\pm 0^{\prime \prime}$ & $45^{\circ}$ & $\pm 0^{\prime \prime}$ & $44^{\circ} 59^{\prime} 59.36^{\prime \prime}$ & $\pm 0.26^{\prime \prime}$ \\
\hline $45^{\circ} 00^{\prime} 00.00^{\prime \prime}$ & $\pm 0^{\prime \prime} 89^{\circ} 00^{\prime} 07.96^{\prime \prime}$ & $\pm 0^{\prime \prime}$ & $45^{\circ}$ & $\pm 0^{\prime \prime} 44^{\circ} 59^{\prime} 59.87^{\prime \prime}$ & $\pm 0.05^{\prime \prime}$ \\
\hline
\end{tabular}

Table 4: Worked examples of reducing measured directions to the ellipsoid (Eq. 11) and error propagation (Eq. 12) using the deflection errors from Appendix A. The [unrealistic] cases of zero observational error are included to assist gauge the relative contribution of the deflection errors.

\subsection{Laplace's equation}

Laplace's equation is used for the conversion from an astronomic / gyrotheodolite azimuth $(A)$ to a geodetic azimuth $(\alpha)$ and vice versa is:

$\alpha=A-\eta \cdot \tan \phi-(\xi \cdot \sin \alpha-\eta \cdot \cos \alpha) \cot z$ 
which for geodetic zenith angles close to $90^{\circ}$ simplifies to

$\alpha \approx A-\eta \cdot \tan \phi$

and the corresponding error is

$\sigma(\alpha) \approx \sqrt{\sigma^{2}(A)+\tan ^{2}(\phi) \sigma^{2}(\eta)+\left(\frac{\eta}{\cos ^{2}(\phi)}\right)^{2} \sigma^{2}(\phi)}$

Equation (13) strictly requires iteration to determine the geodetic azimuth to use in the right hand side. A first approximation is to use the astronomic or gyrotheodolite azimuth. Since the Laplace correction is reasonably small (less than 50" in Australia; cf. Appendix A), one iteration is usually adequate.

\begin{tabular}{|l|r|c|}
\hline \multicolumn{1}{|c|}{ Astronomic/Gyrotheodolite azimuth } & $A=306^{\circ} 43^{\prime} 28.20^{\prime \prime}$ & $\sigma(A)=1^{\prime \prime}$ \\
\hline \multirow{2}{*}{ GDA2020 geodetic coordinates } & $\phi=-25^{\circ} 56^{\prime} 56.86000^{\prime \prime}$ & $\sigma(\phi)=0.00001^{\prime \prime}$ \\
\cline { 2 - 3 } & $\lambda=133^{\circ} 12^{\prime} 37.21000^{\prime \prime}$ & \\
\hline AUSGeoid2020 vertical deflection & $\eta=-7.96^{\prime \prime}$ & $\sigma(\eta)= \pm 3.11^{\prime \prime}$ \\
\hline Geodetic azimuth from Eq. (14) & $\alpha=306^{\circ} 43^{\prime} 24.33^{\prime \prime}$ & $\sigma(\alpha)= \pm 1.81^{\prime \prime}$ \\
\hline
\end{tabular}

Table 5: Worked example of the simplified Laplace's equation (Eq. 14) and error propagation (Eq. 15) using the deflection errors from Appendix A.

\subsection{Transformation between astronomical $(\Lambda, \Phi)$ to geodetic $(\lambda, \phi)$ coordinates}

This transformation is rarely used nowadays because positional astronomy has largely been superseded by satellite positioning systems, so we only include it here for completeness. Pseudo-iteration is also used here to determine approximate geodetic coordinates for determination of the vertical deflections.

$\phi=\Phi-\xi$

$\lambda=\Lambda-\frac{\eta}{\cos (\phi)}$

$\sigma(\phi)=\sqrt{\sigma^{2}(\Phi)+\sigma^{2}(\xi)}$

Due to independence of the deflections assumed by (i) and (ii) above, allows

$\sigma(\lambda)=\sqrt{\sigma^{2}(\Lambda)+\left(\frac{1}{\cos (\phi)}\right)^{2} \sigma^{2}(\eta)+\left(\frac{\eta \tan (\phi)}{\cos (\phi)}\right)^{2} \sigma^{2}(\phi)}$

\begin{tabular}{|c|r|c|}
\hline Astronomical coordinates & $\Phi=-25^{\circ} 56^{\prime} 54.55^{\prime \prime}$ & $\sigma(\Phi)=1^{\prime \prime}$ \\
\cline { 2 - 3 } & $\Lambda=133^{\circ} 12^{\prime} 30.08^{\prime \prime}$ & $\sigma(\Lambda)=1^{\prime \prime}$ \\
\hline AUSGeoid2020 vertical deflections & $\xi=+2.44^{\prime \prime}$ & \\
\cline { 2 - 3 } & $\eta=-7.92^{\prime \prime}$ & \\
\hline Approximate geodetic coordinates & $\phi=-25^{\circ} 56^{\prime} 56.99^{\prime \prime}$ & \\
\cline { 2 - 3 } & $\lambda=133^{\circ} 12^{\prime} 38.89^{\prime \prime}$ & \\
\hline AUSGeoid2020 vertical deflections & $\xi=+2.44^{\prime \prime}$ & $\sigma(\xi)=2.84^{\prime \prime}$ \\
\cline { 2 - 3 } & $\eta=-7.97^{\prime \prime}$ & $\sigma(\eta)=3.11^{\prime \prime}$ \\
\hline GDA2020 geodetic coordinates & $\phi=-25^{\circ} 56^{\prime} 56.99^{\prime \prime}$ & $\sigma(\phi)=3.02^{\prime \prime}$ \\
\cline { 2 - 4 } & $\lambda=133^{\circ} 12^{\prime} 38.94^{\prime \prime}$ & $\sigma(\lambda)=3.60^{\prime \prime}$ \\
\hline
\end{tabular}

Table 6: Worked example of transformation from astronomical to geodetic coordinates

(Eqs. 16 and 17) and error propagation (Eqs. 18 and 19) using the deflection errors from Appendix A.

\section{AUSGeoid2020 effects on geodetic network adjustments}

In this Section, we provide worked examples to demonstrate the effects of AUSGeoid2020 and deflection errors on simulated network adjustments, involving: (i) terrestrial-only observations; (ii) GNSS-only observations; and (iii) a combined terrestrial-GNSS network. For the GNSS-only network, deflections are 
not needed and the separation values are only used in the post-adjustment transformation of GNSS ellipsoidal heights to AHD heights (cf. Eq. 1).

Our simulated terrestrial geodetic network is a small braced quadrilateral of $\sim 2 \mathrm{~km}$ by $\sim 400 \mathrm{~m}$ (Figure 1). The input data are shown in Tables 7 and 8 . Network (i) contains the following simulated observations: horizontal directions, zenith angles, and EDM-measured slope distances from a total station survey, and height differences from differential levelling. Observations from each station to each of the three remaining stations were simulated, hence there are a total of 12 observations of each type. Network (ii) contains six independent simulated baselines (3D coordinate differences) between each pair of stations. Network (iii) contains all of the observations from networks (i) and (ii) combined into one.

All observations are simulated to be error-free, but variances are applied based upon 'typical' manufacturer-stated instrument precisions: i.e., horizontal directions \pm 1 ", zenith angles \pm 2 ", calibrated EDM slope distances ( $\pm 2 \mathrm{~mm} \pm 2 \mathrm{ppm})$, differential levelling ( $\pm 12 \sqrt{d} \mathrm{~mm}$, where $d$ is distance in $\mathrm{km}), \mathrm{GNSS}$ baselines ( $\pm 10 \mathrm{~mm} \pm 1 \mathrm{ppm}$ ), centring errors $\pm 1.5 \mathrm{~mm}$, instrument, antenna and target height errors $\pm 1 \mathrm{~mm}$.

Firstly, the effect of neglecting the uncertainty in AUSGeoid2020 and vertical deflections in a 3D minimally constrained adjustment of the three survey networks is investigated. For this purpose, the three networks were adjusted under two different scenarios: (1) the simulated observations and all AUSGeoid2020 values for full reduction to the GDA2020 are included, but without uncertainty estimates for GDA2020-AHD separations and deflections (i.e., these are considered error-free), and (2) the simulated observations and AUSGeoid2020 values with uncertainty estimates for GDA2020-AHD separations and deflections uncertainties (Appendix A) included. For the GNSS-only and combined networks, the coordinates of station MRE were held fixed, while for the terrestrial-only network, the geodetic azimuth from station MRE to station P189 was also held fixed.

Beforehand however, it is instructive to place a caveat emptor on least squares adjustment packages because separations and deflections are generally to be considered error-free, whereas they are not. In these worked examples, we use the MOVE3 least squares adjustment software (free demonstration version: https://move3software.com/free-demo/). The input and output files are provided as Electronic Supplementary Material to this article for replication.

In MOVE3, a single network-wide separation uncertainty can be included, but there is [yet] no option to include location-specific geoid uncertainty values. Thus, a mean separation uncertainty of \pm 0.082 $\mathrm{m}$ (cf. Table 8) was included in these simulations. MOVE3 also has no direct option to include uncertainties in vertical deflections. Therefore, the influence of deflection uncertainty on the zenith angle uncertainty (Eq. 10) was calculated for each zenith angle individually and per azimuth, and then inserted into MOVE3 manually.

Table 9 shows the differences in the resulting uncertainties in station coordinates between cases (1) treating the separations and deflections as error-free, and (2) including separation and deflection uncertainties. Only the uncertainty of the derived AHD heights is influenced by the separation and deflection uncertainties. In the terrestrial-only network (i) and the combined network (iii), the inclusion of deflection uncertainties causes a moderate increase in the standard deviations of the heights of the stations, while the separation uncertainty is irrelevant. In the GNSS-only network (ii), the inclusion of separation uncertainty causes a large increase in the standard deviations of the heights of the stations.

Finally, we also investigate a further three extremely wrong scenarios where AUSGeoid2020 values are not included in the adjustment: (3) no separations nor deflections, (4) no separations but deflections, and (5) no deflections but separations.Table 10 summarises the errors in the coordinates of station STN4 (the station furthest from the fixed station MRE) for these three additional cases. The absence of deflections has only a minor effect on the terrestrial-only network (i) because the deflections are small in magnitude (Table 8). The absence of separations has the most significant effect on the heights of stations in the GNSS-only network (ii), but it also has a significant effect on the horizontal coordinates in all three networks. A final point to note is that the error in the height in the terrestrial-only network (i) is $-0.015 \mathrm{~m}$ when only the separations are excluded (case 4), but an order of magnitude smaller when the deflections are also excluded (case 3 ). 


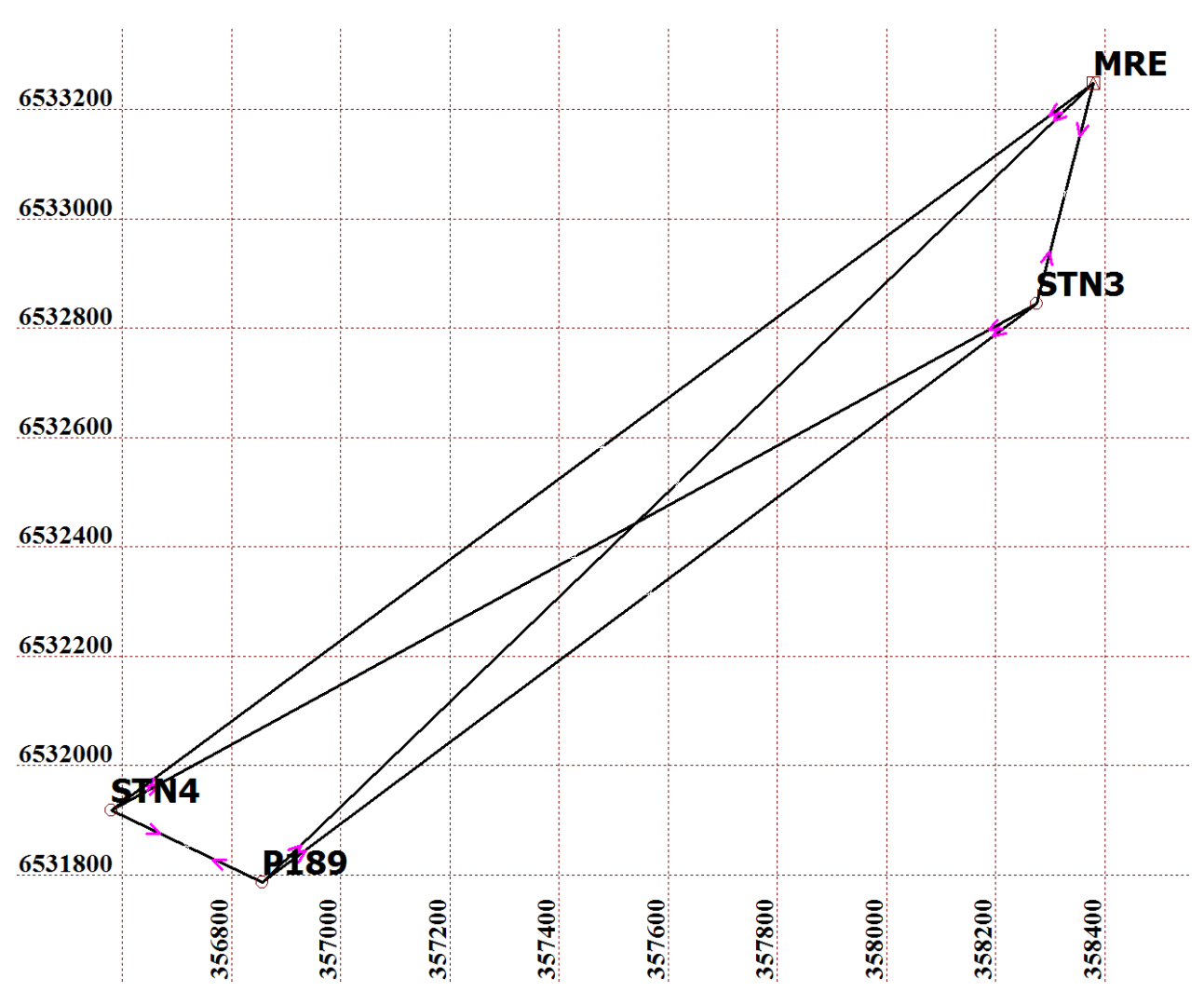

Figure 1: Simulated network (MGA2020 zone 50S coordinates).

\begin{tabular}{|l|r|r|r|}
\hline \multirow{2}{*}{ Station } & \multicolumn{2}{|c|}{ GDA2020 } & \multicolumn{1}{c|}{ AHD } \\
\cline { 2 - 4 } & Longitude & Latitude & Height $(\mathrm{m})$ \\
\hline MRE & $115^{\circ} 30^{\prime} 41.45561^{\prime \prime}$ & $-31^{\circ} 19^{\prime} 35.62740^{\prime \prime}$ & 71.108 \\
\hline P189 & $115^{\circ} 29^{\prime} 43.10817^{\prime \prime}$ & $-31^{\circ} 20^{\prime} 22.39007^{\prime \prime}$ & 56.081 \\
\hline STN3 & $115^{\circ} 30^{\prime} 37.35105^{\prime \prime}$ & $-31^{\circ} 19^{\prime} 48.64830^{\prime \prime}$ & 52.069 \\
\hline STN4 & $115^{\circ} 29^{\prime} 32.71017^{\prime \prime}$ & $-31^{\circ} 20^{\prime} 18.01215^{\prime \prime}$ & 41.944 \\
\hline
\end{tabular}

Table 7: Station coordinates in the simulated network

\begin{tabular}{|l|r|r|r|r|r|r|}
\hline Station & $N(m)$ & $\eta$ & $\xi$ & $\sigma(N)(m)$ & $\sigma(\eta)$ & $\sigma(\xi)$ \\
\hline MRE & -32.361 & $-4.42^{\prime \prime}$ & $0.34^{\prime \prime}$ & \pm 0.081 & $\pm 3.06^{\prime \prime}$ & $\pm 3.51^{\prime \prime}$ \\
\hline P189 & -32.397 & $-5.10^{\prime \prime}$ & $-0.12^{\prime \prime}$ & \pm 0.083 & $\pm 3.05^{\prime \prime}$ & $\pm 3.47^{\prime \prime}$ \\
\hline STN3 & -32.370 & $-4.61^{\prime \prime}$ & $0.26^{\prime \prime}$ & \pm 0.081 & $\pm 3.05^{\prime \prime}$ & $\pm 3.50^{\prime \prime}$ \\
\hline STN4 & -32.394 & $-5.16^{\prime \prime}$ & $-0.09^{\prime \prime}$ & \pm 0.083 & $\pm 3.05^{\prime \prime}$ & $\pm 3.47^{\prime \prime}$ \\
\hline
\end{tabular}

Table 8: AUSGeoid2020 input data for the simulated network.

\begin{tabular}{|l|c|c|c|c|c|c|c|}
\hline & Station & \multicolumn{3}{|c|}{ Case 1 } & \multicolumn{3}{c|}{ Case 2 } \\
\cline { 2 - 7 } & & $\sigma($ East) & $\sigma$ (North) & $\sigma$ (Height) & $\sigma($ East $)$ & $\sigma($ North) & $\sigma$ (Height) \\
\hline \multirow{4}{*}{ (i) Terrestrial-only } & P189 & \pm 0.0024 & \pm 0.0024 & \pm 0.0048 & \pm 0.0024 & \pm 0.0024 & \pm 0.0060 \\
\cline { 2 - 7 } & STN3 & \pm 0.0020 & \pm 0.0023 & \pm 0.0025 & \pm 0.0020 & \pm 0.0023 & \pm 0.0036 \\
\cline { 2 - 7 } & STN4 & \pm 0.0028 & \pm 0.0032 & \pm 0.0048 & \pm 0.0028 & \pm 0.0032 & \pm 0.0060 \\
\hline (ii) GNSS-only & P189 & \pm 0.0083 & \pm 0.0083 & \pm 0.0083 & \pm 0.0083 & \pm 0.0083 & \pm 0.0586 \\
\cline { 2 - 7 } & STN3 & \pm 0.0080 & \pm 0.0080 & \pm 0.0079 & \pm 0.0080 & \pm 0.0080 & \pm 0.0585 \\
\cline { 2 - 7 } & STN4 & \pm 0.0084 & \pm 0.0084 & \pm 0.0083 & \pm 0.0084 & \pm 0.0084 & \pm 0.0586 \\
\hline (iii) Combined & P189 & \pm 0.0048 & \pm 0.0050 & \pm 0.0040 & \pm 0.0048 & \pm 0.0050 & \pm 0.0059 \\
\cline { 2 - 7 } & STN3 & \pm 0.0023 & \pm 0.0022 & \pm 0.0024 & \pm 0.0023 & \pm 0.0022 & \pm 0.0036 \\
\cline { 2 - 7 } & STN4 & \pm 0.0045 & \pm 0.0058 & \pm 0.0040 & \pm 0.0045 & \pm 0.0058 & \pm 0.0060 \\
\hline
\end{tabular}


Table 9: Standard deviations of station coordinates [in metres] for cases:

(1) separations and deflections considered error-free, and

(2) AUSGeoid2020 separation and deflection uncertainties included.

\begin{tabular}{|l|c|c|c|c|c|c|c|c|r|}
\hline & \multicolumn{3}{|c|}{ Case 3 } & \multicolumn{3}{c|}{ Case 4 } & \multicolumn{3}{c|}{ Case 5 } \\
\cline { 2 - 11 } & East & North & Height & East & North & Height & East & North & Height \\
\hline (i) Terrestrial-only & 0.010 & 0.005 & -0.002 & 0.009 & 0.007 & -0.015 & 0.001 & -0.002 & -0.002 \\
\hline (ii) GNSS-only & 0.009 & 0.007 & -0.033 & 0.009 & 0.007 & -0.033 & 0.000 & 0.000 & 0.000 \\
\hline (iii) Combined & 0.009 & 0.006 & -0.012 & 0.009 & 0.007 & -0.021 & 0.000 & 0.000 & -0.001 \\
\hline
\end{tabular}

Table 10: Errors [in metres] in the MGA2020 Easting, Northing and AHD height of station STN4 due to (3) no separations and deflections, (4) no separations but deflections, and (5) no deflections but separations.

\section{Concluding remarks and recommendations}

We have given a series of worked examples on the use of AUSGeoid2020 GDA2020-AHD separations and vertical deflections in the reduction and least squares adjustment of geodetic surveying data. We have also derived some new error-propagation formulas for the more common geodetic surveying reductions. AUSGeoid2020 GDA2020-AHD separations are already accompanied by a grid of error estimates, so here we provide new grids of error estimates of the EW and NS vertical deflections (Appendix A). All are provided at https://s3-ap-southeast-2.amazonaws.com/geoid/.

The error propagation formulas for vertical deflections are sometimes non-linear, so the errors from the vertical deflection errors will propagate differently depending on the configuration of the geodetic surveys. For instance, the error propagation for the reduction of zenith angles varies as a function of azimuth (Section 3.1), and the error propagation for the reduction of horizontal directions varies as a function of both azimuth and zenith angle (Section 3.2), where directions observed for zenith angles less than 5 degrees in magnitude from the horizon significantly diminish the effect of vertical deflection errors on the survey. Therefore, use of these error propagation formulas should assist geodetic surveyors in their observational design so as to be less exposed to uncertainties in vertical deflections.

Most public-domain and commercially available least squares adjustment packages do not permit error estimates for the geoid-ellipsoid separation and vertical deflections, instead treating them as known or fixed values, i.e., error-free. We therefore recommend that software developers add such functionality so that geodetic surveyors who wish to consider the propagation these errors to their results may do so, instead of the somewhat cumbersome technique we had to use in Section 4.

\section{Appendix A: Error grids for vertical deflections}

The AUSGeoid2020 vertical deflections in the meridian NS $(\xi)$ and prime vertical EW $(\eta)$ are determined from the gravimetric AGQG2017 using the following formulas. Grids of these values are provided at https://s3-ap-southeast-2.amazonaws.com/geoid/.

$$
\begin{aligned}
& \xi_{G}(\phi, \lambda)=\arctan \left(\frac{\zeta(\phi+\Delta \phi, \lambda)-\zeta(\phi-\Delta \phi, \lambda)}{2 \rho \Delta \phi}\right) \\
& \eta_{G}(\phi, \lambda)=\arctan \left(\frac{\zeta(\phi, \lambda+\Delta \lambda)-\zeta(\phi, \lambda-\Delta \lambda)}{2 v \Delta \lambda \cos \phi}\right)
\end{aligned}
$$

where the meridional radius of curvature of the ellipsoid is

$$
\rho=\frac{a\left(1-e^{2}\right)}{\left(1-e^{2} \sin ^{2} \phi\right)^{3 / 2}}
$$

and the prime vertical radius of curvature of the ellipsoid is

$$
v=\frac{a}{\left(1-e^{2} \sin ^{2} \phi\right)^{1 / 2}}
$$

The GDA2020, AGQG2017 and AUSGeoid2020 all use the GRS80 ellipsoid. 
Using the uncertainty variance of the difference between two AUSGeoid2020 values from Eq. (8), i.e., $\left\{\sigma^{2}\left(\zeta_{1}\right)+\sigma^{2}\left(\zeta_{2}\right)\right\}\left(1-k \exp ^{-3 l / a}\right)$ noting the use of the quasigeoid here, the standard deviation of the vertical deflections can be approximated (to first order) by

$$
\begin{aligned}
\sigma\left(\xi_{G}\right) & =\left(\frac{2 \rho \Delta \phi}{(2 \rho \Delta \phi)^{2}+(\zeta(\phi+\Delta \phi, \lambda)-\zeta(\phi-\Delta \phi, \lambda))^{2}}\right) \\
& \times \sqrt{\operatorname{var}(\zeta(\phi+\Delta \phi, \lambda)-\zeta(\phi-\Delta \phi, \lambda))} \\
= & \left(\frac{2 \rho \Delta \phi}{(2 \rho \Delta \phi)^{2}+(\zeta(\phi+\Delta \phi, \lambda)-\zeta(\phi-\Delta \phi, \lambda))^{2}}\right) \\
& \times \sqrt{\left\{\sigma^{2}(\zeta(\phi+\Delta \phi, \lambda))+\sigma^{2}(\zeta(\phi-\Delta \phi, \lambda))\right\}\left(1-\rho \exp ^{-3 l / a}\right)} \\
\sigma\left(\eta_{G}\right)= & \left(\frac{2 v \Delta \lambda \cos \phi}{(2 v \Delta \lambda \cos \phi)^{2}+(\zeta(\phi, \lambda+\Delta \lambda)-\zeta(\phi, \lambda-\Delta \lambda))^{2}}\right) \\
& \times \sqrt{\operatorname{var}(\zeta(\phi, \lambda+\Delta \lambda)-\zeta(\phi, \lambda-\Delta \lambda))} \\
= & \left(\frac{2 v \Delta \lambda \cos \phi}{(2 v \Delta \lambda \cos \phi)^{2}+(\zeta(\phi, \lambda+\Delta \lambda)-\zeta(\phi, \lambda-\Delta \lambda))^{2}}\right) \\
& \times \sqrt{\left\{\sigma^{2}(\zeta(\phi, \lambda+\Delta \lambda))+\sigma^{2}(\zeta(\phi, \lambda-\Delta \lambda))\right\}\left(1-\rho \exp ^{-3 l / a}\right)}
\end{aligned}
$$

Figure $A 1$ shows the vertical deflections (Eqs. A1 \& A2) and their uncertainties (Eqs. A5 \& A6).

Due to the way in which we have modelled the covariance of the quasigeoid uncertainties (i.e., Eq. A7), the covariance of the uncertainty between $\xi$ and $\eta$ is zero, as follows.

$$
\begin{aligned}
\operatorname{cov}\left(\zeta_{1}, \zeta_{2}\right) & =\left\{\sigma^{2}\left(\zeta_{1}\right)+\sigma^{2}\left(\zeta_{2}\right)\right\} \frac{k}{2} \exp ^{-3 l / a} \\
\operatorname{cov}(\xi, \eta) & =\left(\frac{2 \rho \Delta \phi}{(2 \rho \Delta \phi)^{2}+(\zeta(\phi+\Delta \phi, \lambda)-\zeta(\phi-\Delta \phi, \lambda))^{2}}\right) \\
& \times\left(\frac{2 v \Delta \lambda \cos \phi}{(2 v \Delta \lambda \cos \phi)^{2}+(\zeta(\phi, \lambda+\Delta \lambda)-\zeta(\phi, \lambda-\Delta \lambda))^{2}}\right) \\
& \times \operatorname{cov}\{\zeta(\phi, \lambda+\Delta \lambda)-\zeta(\phi, \lambda-\Delta \lambda), \zeta(\phi+\Delta \phi, \lambda)-\zeta(\phi-\Delta \phi, \lambda)\} \\
\operatorname{cov}(\xi, \eta)= & \left(\frac{2 \rho \Delta \phi}{(2 \rho \Delta \phi)^{2}+(\zeta(\phi+\Delta \phi, \lambda)-\zeta(\phi-\Delta \phi, \lambda))^{2}}\right) \times\left(\frac{\text { A7) }}{(2 v \Delta \lambda \cos \phi)^{2}+(\zeta(\phi, \lambda+\Delta \lambda)-\zeta(\phi, \lambda-\Delta \lambda))^{2}}\right) \\
\times & \left\{\begin{array}{l}
\sigma^{2}(\zeta(\phi, \lambda+\Delta \lambda))+\sigma^{2}(\zeta(\phi+\Delta \phi, \lambda))-\sigma^{2}(\zeta(\phi, \lambda+\Delta \lambda)) \\
-\sigma^{2}(\zeta(\phi-\Delta \phi, \lambda))-\sigma^{2}(\zeta(\phi, \lambda-\Delta \lambda))-\sigma^{2}(\zeta(\phi+\Delta \phi, \lambda)) \\
-\sigma^{2}(\zeta(\phi, \lambda-\Delta \lambda))+-\sigma^{2}(\zeta(\phi-\Delta \phi, \lambda))
\end{array}\right) \\
\times & \frac{k}{2} \exp \left(\frac{-3 \sqrt{(2 \rho \Delta \phi)^{2}+(2 v \Delta \lambda \cos \phi)^{2}}}{a}\right)=0
\end{aligned}
$$

where the variance terms $\sigma^{2}$ between the grid nodes all cancel to zero. As such, we do not need to $\operatorname{consider} \operatorname{cov}(\xi, \eta)$ in any of the equations listed in the main text. 

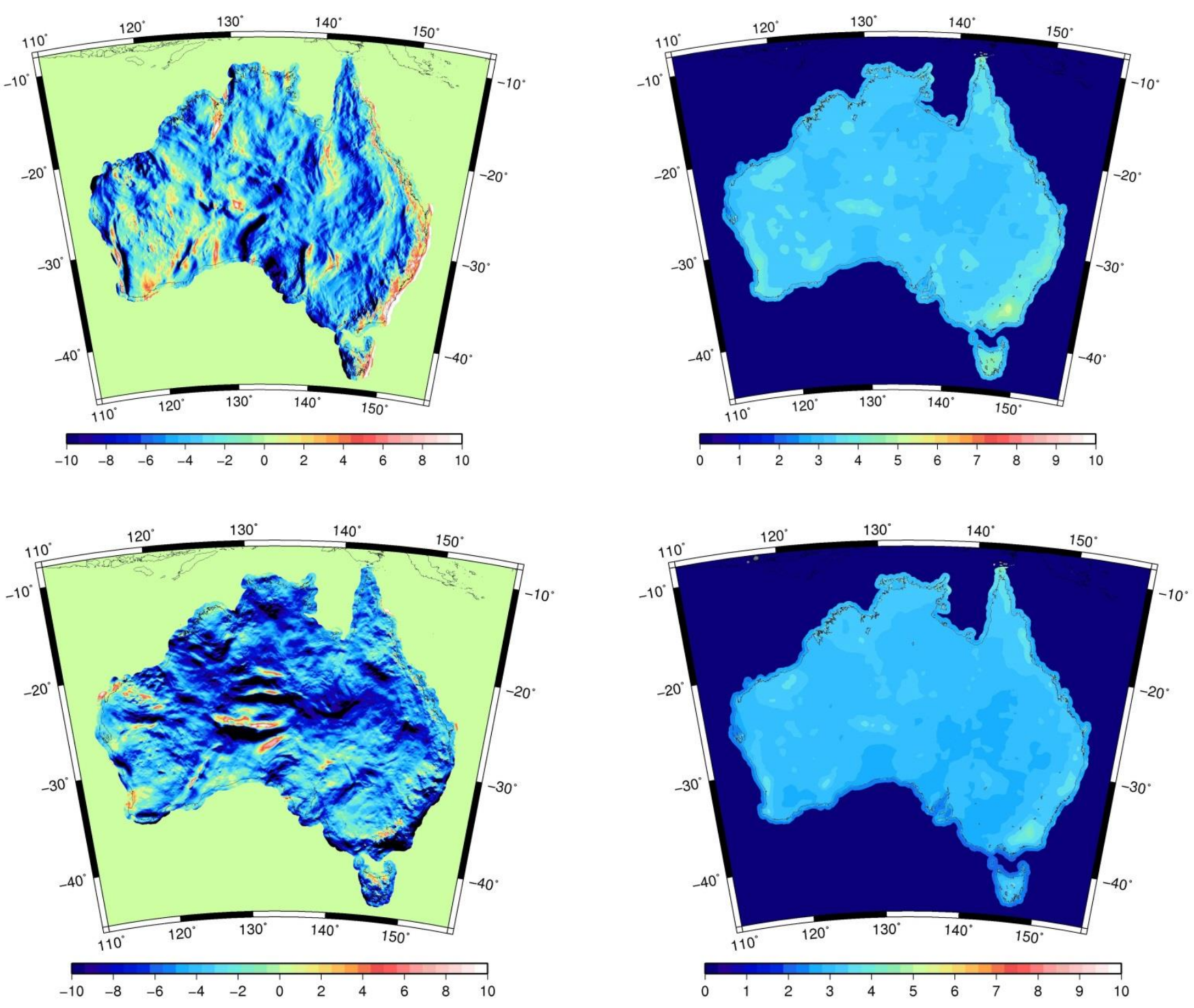

Figure A1: AGQG2017-derived Pizzetti vertical deflections [in arc-seconds]

(a) in the prime vertical [min: $-41.06^{\prime \prime} \max :+40.55^{\prime \prime}$, mean: $-0.63^{\prime \prime}$ STD: $\pm 2.15^{\prime \prime}$;

(b) in the meridian [min: $-47.55^{\prime \prime}$ max: $31.89^{\prime \prime}$, mean: $-0.96^{\prime \prime}$ STD: $\left.\pm 2.50^{\prime \prime}\right]$;

(c) prime vertical error standard deviations [min: 0" max: 6.50", mean: 0.61" STD: $\pm 1.27^{\prime \prime}$;

(d) meridional error standard deviations [min: 0" max: 6.45", mean: 0.55" STD: $\pm 1.16^{\prime \prime}$ ]

\section{Acknowledgements}

We would like to thank the two anonymous reviewers for their extremely prompt and constructive reviews of an earlier version of this manuscript. Jack McCubbine and Nicholas Brown publish this paper with the permission of the Chief Executive Officer of Geoscience Australia.

\section{References}

Brown, N.J., W.E. Featherstone, G. Hu and G.M. Johnston (2011) AUSGeoid09: a more direct and more accurate model for converting ellipsoidal heights to AHD heights, Journal of Spatial Science 56(1): 27-37, https://doi.org/10.1080/14498596.2011.580498

Brown, N.J., J.C. McCubbine, W.E. Featherstone, N. Gowans, A. Woods and I. Baran (2018a) AUSGeoid2020 combined gravimetric-geometric model with location-specific uncertainties and baseline-length-dependent error decorrelation, Journal of Geodesy, https://doi.org/10.1007/s00190-018-1202-7.

Brown, N.J., J.C. McCubbine, W.E. Featherstone, N. Gowans, A. Woods and I. Baran (2018b) Correction to: AUSGeoid2020 combined gravimetric-geometric model with location-specific uncertainties and baseline-lengthdependent error decorrelation, Journal of Geodesy, https://doi.org/10.1007/s00190-018-1214-3. 
Dow, J.M., R.E. Neilan and C. Rizos (2009) The International GNSS Service in a changing landscape of Global Navigation Satellite Systems, Journal of Geodesy 83(3-4): 191-198, https://doi.org/10.1007/s00190-008-0300-3.

Featherstone, W.E. (1997) The importance of including the geoid in terrestrial survey data reduction to the Geocentric Datum of Australia, The Australian Surveyor 42(1): 45-50. https://doi.org/10.1080/00050342.1997.10558665

Featherstone, W.E. (1998) Do we need a gravimetric geoid or a model of the base of the Australian Height Datum to transform GPS heights? The Australian Surveyor 43(4): 273-280. https://doi.org/10.1080/00050350.1998.10558758

Featherstone, W.E. (2008) GNSS-based heighting in Australia: current, emerging and future issues, Journal of Spatial Science 53(2): 115-133, https://doi.org/10.1080/14498596.2008.9635153

Featherstone, W.E. and J.M. Rüeger (2000) The importance of using deviations of the vertical in the reduction of terrestrial survey data to a geocentric datum, The Trans-Tasman Surveyor 1(3): 46-61. [Erratum in The Australian Surveyor 47(1): 7] https://doi.org/10.1080/00050354.2000.10558815

Featherstone, W.E. and M. Kuhn (2006) Height systems and vertical datums: a review in the Australian context, Journal of Spatial Science 51(1): 21-42, https://doi.org/10.1080/14498596.2006.9635062

Featherstone, W.E. and M.S. Filmer (2012) The north-south tilt in the Australian Height Datum is explained by the ocean's mean dynamic topography, Journal of Geophysical Research - Oceans 117(C8), C08035, https://doi.org/10.1029/2012JC007974.

Featherstone, W.E., J.C. McCubbine, N.J. Brown, S.J. Claessens, M.S. Filmer, and J.F. Kirby (2018) The first Australian gravimetric quasigeoid model with location-specific uncertainty estimates, Journal of Geodesy 92(2): 149-168, https://doi.org/10.1007/s00190-017-1053-7

Featherstone, W.E., J.F. Kirby, C. Hirt, M.S. Filmer, S.J. Claessens, N.J. Brown, G. Hu and G.M. Johnston (2011) The AUSGeoid09 model of the Australian Height Datum, Journal of Geodesy 85(3): 133-150, https://doi.org/10.1007/s00190-010-0422-2

Hirt, C., M.S. Filmer and W.E. Featherstone (2010) Comparison and validation of recent freely-available ASTER-GDEM ver1, SRTM ver4.1 and GEODATA DEM-9S ver3 digital elevation models over Australia, Australian Journal of Earth Sciences 57(3): 337-347, https://doi.org/10.1080/08120091003677553

ICSM (2018) Geocentric Datum of Australia 2020 Technical Manual (version 1.1.1), Inter-governmental Committee on Surveying and Mapping, Canberra, http://www.icsm.gov.au/sites/default/files/GDA2020TechnicalManualV1.1.1.pdf

Jekeli C (1999) An analysis of vertical deflections derived from high-degree spherical harmonic models. Journal of Geodesy 73(1): 10-22. https://doi.org/10.1007/s001900050213.

Kearsley, A.H.W. (1986) Data requirements for determining precise relative geoid heights from gravimetry. Journal of Geophysical Research 91(89): 9193-9201. https://doi.org/10.1029/JB091iB09p09193

Kearsley, A.H.W. (1988) Tests on the recovery of precise geoid height differences from gravimetry. Journal of Geophysical Research 93(B6): 6559-6570. https://doi.org/10.1029/JB093iB06p06559

Mather, R.S. (1970) The geocentric orientation vector for the Australian Geodetic Datum, Geophysical Journal of the Royal Astronomical Society 22(1): 55-81, https://doi.org/10.1111/j.1365-246X.1971.tb03583.x

Rothacher, M. (2002) Estimation of station heights with GPS, in: Drewes, H., Dodson, A., Fortes, L.P.S., Sanchez, L. and Sandoval, P. (Eds.), Vertical Reference Systems, International Association of Geodesy Symposia 124, Springer, Berlin, Heidelberg, pp. 81-90. https://doi.org/10.1007/978-3-662-04683-8 17.

Rüeger, J.M. (1996) Electronic Distance Measurement - An Introduction (fourth edition), Springer, Berlin-HeidelbergNew York. 